\title{
Searching for the origin of the enigmatic circulating T follicular helper cells
}

\author{
Savita Pahwa \\ Department of Microbiology and Immunology, University of Miami Miller School of Medicine, Miami, Florida, USA.
}

T follicular helper (Tfh) cells in germinal centers of secondary lymphoid organs are pivotal for $B$ and $T$ cell interactions required for induction of humoral immunity. It has long been debated whether Tfh cells exit from lymph nodes into the blood as circulating Tfh cells. In this issue of the $J C I$, Vella et al. have taken the bull by the horns and applied considerable technical muscle to answer this question. By analyzing phenotype, transcriptome, epigenetic profile, and T cell receptor clonotype, the authors provide evidence that a subset of cTfh cells do indeed originate in lymph nodes and traffic into the blood via the thoracic duct.

\section{A cell type of mysterious origin}

The center of antibody production lies in lymphoid germinal centers (GCs), the seat of the $\mathrm{T}$ follicular helper (Tfh) cell interaction with B cells. Here, Tfh cells, a distinct subset of specialized $\mathrm{CD}^{+} \mathrm{T}$ cells, help $\mathrm{B}$ cells to undergo proliferation, isotype switching, and somatic hypermutation to form high-affinity antibodies $(1,2)$. Our understanding of lymphoid Tfh cells is derived largely from studies in mice. Surgical human lymph node biopsies for research are not practical; however, recent studies suggest that fine-needle aspiration of lymph nodes (3) could prove to be a promising alternative. The discovery of lymphocytes with Tfh-like properties in human peripheral blood (4) naturally sparked a new era of investigation by researchers hoping that this circulating population may be a more accessible record of events in secondary lymphoid organs (SLOs; refs. 5, 6). But conclusive proof of a lymphoid origin for circulating Tfh (cTfh) cells has been lacking.

\section{Tfh cell development and} traffic in lymph nodes

Lymphocyte traffic to and from SLOs has intrigued and fascinated immunologists for decades. Sir James Gowans demonstrated in 1964 that rat lymphocytes circulate from blood to lymphoid organs one to two times a day (7). Lymphocytes are transported into lymph nodes via afferent lymphatic vessels in lymph that percolates through the subcapsular, medullary, and cortical sinuses. They exit via efferent lymphatic vessels that merge into the thoracic duct, which empties into the subclavian vein (8). Another portal for entry of lymphocytes into SLO is via high endothelial venules, which are specialized blood vessels in the paracortex (8), suggesting a plausible vascular route of lymphoid cell egress from SLO. Cues that regulate lymphocyte entry, retention, and exit from SLO are not fully understood and are regulated by a number of receptor/ligand interactions and gradients $(9,10)$. Key examples are CCR7/CCL21 for lymphocyte homing into lymph nodes, CXCR5/CCL13 for follicular trafficking of CXCR5 ${ }^{+}$Tfh cells, and sphingosine-1-phosphate receptor 1 (SIPR1)/SIP for lymphocyte egress into efferent lymphatics that contain high concentrations of SIP. Cell-intrinsic circadian clocks and adrenergic nerve activity also influence lymphocyte retention in SLO (11).

\section{Related Article: p. 3185}

Conflict of interest: The author declares that no conflict of interest exists.

Inside the lymph node, $\mathrm{CD}^{+} \mathrm{T}$ cells that are activated by DCs in the $\mathrm{T}$ cell zone acquire distinct Tfh-associated characteristics $\left(\mathrm{CXCR} 5^{+} \mathrm{CCR}^{\mathrm{lo}}{ }^{\mathrm{PD}}-1^{+} \mathrm{ICOS}^{+}\right.$, high BCL-6), as they embark on a purposeful journey with the sole commitment of helping B cells, each step being dependent upon cognate interaction with $\mathrm{B}$ cells. Migrating to the border of the B cell follicle, Tfh cells uniquely cross into the follicle and ultimately engage in the GC reaction as effector Tfh cells. Thereafter, the Tfh might die, revert to memory cells that remain in the same follicle, or traffic out as memory Th cells to adjacent follicles. A fourth possibility, investigated by Vella et al. in this issue (12), is that GC Tfh cells exit out of the lymph node via the thoracic duct into the circulation.

\section{Proving the origin of cTfh cells}

The first step by Vella et al was to establish distinct phenotypic identities of lymphoid Tfh cells as total Tfh cells (CXCR5 ${ }^{+} \mathrm{PD}-1^{+}$), and GC Tfh cells (CXCR5 ${ }^{\text {bright }}$ PD- $1^{\text {bright }}$, herein referred to as $\mathrm{Br} / \mathrm{Br}$ ), which were gated from a nonnaive lymphoid $\mathrm{CD}^{+} \mathrm{T}$ cell population inclusive of memory and effector $\mathrm{CD} 4^{+} \mathrm{T}$ cells. A unique component of this study was the analysis of lymph obtained by cannulation of the thoracic duct of human volunteers. They found that frequency of total and $\mathrm{Br} / \mathrm{Br}$ Th cells was higher in SLO than thoracic duct lymphocytes (TDL) and lowest in blood (Figure 1). The study cautions that tonsils differ from other SLO in frequencies of Tfh cells and shows feasibility of the nonhuman primate model in this type of research.

Abundant data were generated by Vella et al. (12) to support the relationship of $\mathrm{Br} / \mathrm{Br}$ Tfh cells in TDL with GC Tfh cells in SLO. Notably, the overall phenotypic profile of TDL Tfh cells was similar to that of lymph node Tfh cells, with the exception of chemokine receptors CXCR3, CCR6, and CCR4 and the mucosal-trafficking integrin $a_{4} b_{7}$, which were all higher in thoracic duct Tfh cells, as expected for cells required to 


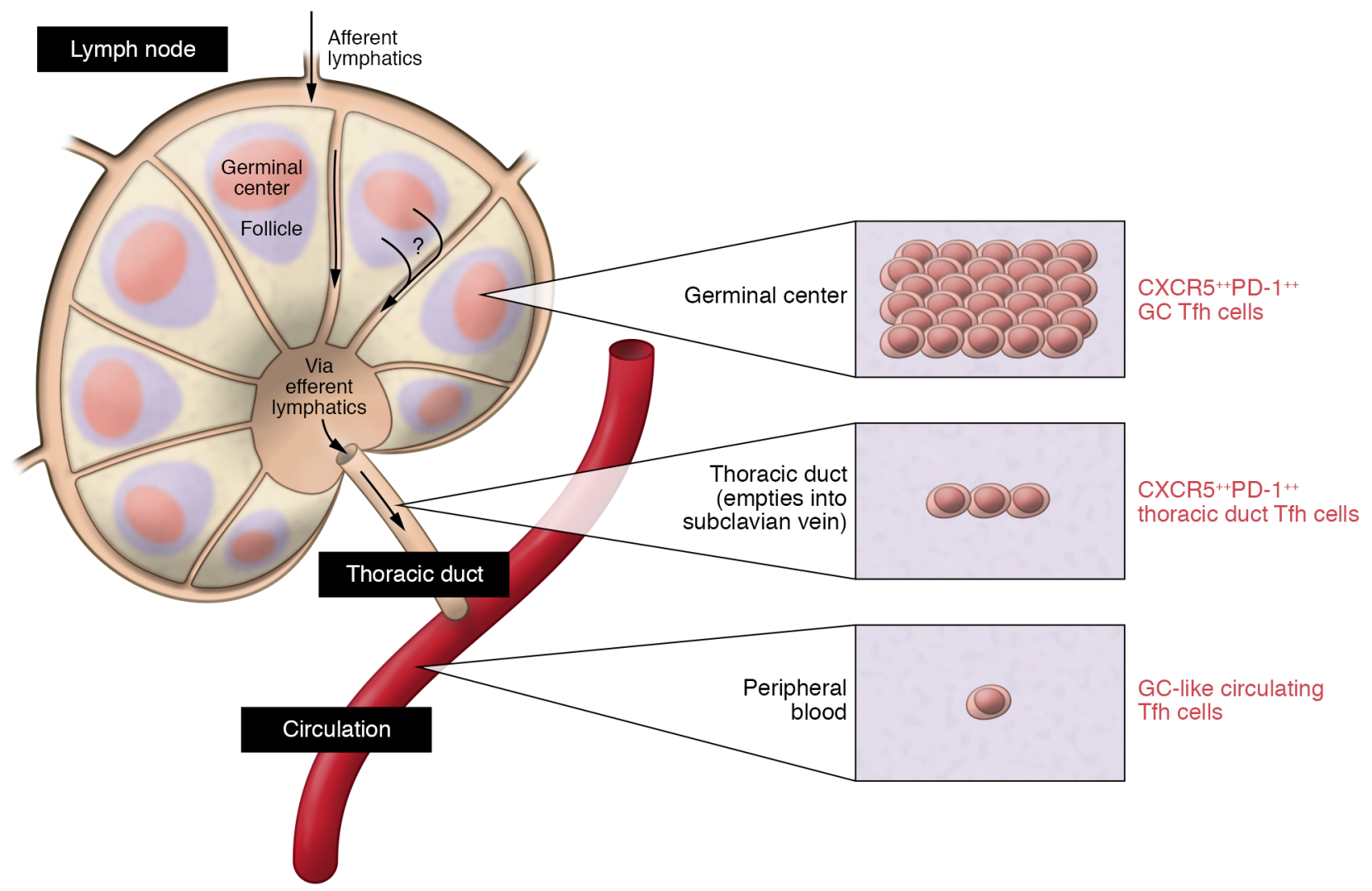

Figure 1. Schematic representation of GC Tfh cells in lymph nodes, thoracic duct, and circulation. Tfh cells in B cell follicles of lymphoid tissue traffic to the GCs, where they provide critical help for B cells. In this issue, Vella et al. identify a population of Tfh cells with high expression of CXCR5 and PD-1 $\left(\mathrm{CXCR}^{++} \mathrm{PD}-1^{++}\right)$that is present in both the GC and thoracic duct and has similar transcriptional and phenotypic characteristics in both locations; however, this population was present at a lower frequency in the thoracic duct than in the lymph nodes. cTfh cells with a similar phenotypic profile were also detectable in blood, but in even lower frequencies. An activated subset (ICOS+CD38 $)$ of cTfh cells that are believed to be vaccine responsive, contained cells with similarities to thoracic duct and lymphoid GC Tfh cells. These studies provide evidence that a population of circulatory Tfh cells originated in the GC of lymph nodes, thereby connecting the biology of Tfh cells in blood to Tfh cells in lymphoid tissue.

migrate to various tissues. Compared with GC Tfh cells, S1PR1 was highly expressed in TDL $\mathrm{Br} / \mathrm{Br}$ Tfh cells, while CD69, a marker of tissue residency and a negative regulator of S1PR1, was downregulated (13), implying egress from SLO into the thoracic duct. Future studies may benefit by taking into consideration the impact of circadian cycles that also influence lymphocyte retention, such as by $\beta 2$ adrenergic receptor-mediated signals (11).

Transcriptional and epigenetic profiling of Tfh cells in different compartments further established the similarity between TDL Br/Br Tfh cells and lymph node GC Tfh cells. Both cell types displayed a common core transcriptional program, with an enrichment of tissue egress-associated genes, such as those encoding S1PR1, S1PR4, and Kruppel-like factor 2 in TDL Tfh cells. Epigenetic analyses also identified commonalities between the two cell types, with changes in locus accessibility that correlated with transcription, and evidence of tonsillar GC Tfh cell signature in both. Importantly, chromatin regions of genes associated with Tfh cell function, such as IL-21 or CXCL13, were more accessible in lymph node GC Tfh cells and TDL $\mathrm{Br} / \mathrm{Br}$ Tfh cells than in any of the other Tfh subsets evaluated. Further transcriptomic and epigenetic studies of known egress pathways and chemokine pathways in defined Tfh cell subsets could yield additional clues into how balance between genes involved in instructing GC Tfh cell trafficking is regulated; accessible open chromatin regions on particular genes could steer the balance between signals that regulate retention versus egress.

A dramatic reduction of circulating $\mathrm{CXCR}^{+} \mathrm{CD}^{+} \mathrm{T}$ cells was observed in patients given the S1PR inhibitor fingolimod (FTY720), supporting the concept of
Tfh cell egress from SLO to populate and sustain the cTfh cells. Establishing the link of the cTfh cells to GC Tfh cells, however, was more challenging. First, the extreme paucity and even absence of $\mathrm{Br} / \mathrm{Br}$ cTfh cells in the blood was unexpected and was attributed mainly to a shortened residency time calculated as one-tenth that of circulating $\mathrm{CD}^{+} \mathrm{T}$ cells. Second, cTfh cells resembled TDL dim/dim Tfh cells rather than $\mathrm{Br} / \mathrm{Br}$ Tfh cells in their transcriptomic and epigenetic profiles and TCR clonotype, implying that a majority of cTfh cells were recirculating resting cells. It was the observation that the TCR sequences of TDL Br/Br Tfh cells overlapped with the TCR of cTfh cells far more than with other TDL Tfh subsets that provided the clue supporting entry of $\mathrm{Br} / \mathrm{Br}$ Tfh cells from TDL into the pool of cTfh cells.

To strengthen evidence for presence of GC cTfh cells in blood, Vella et al. (12) 
Table 1. Conceptualized markers for GC Tfh cells in lymph nodes, GC-derived Tfh cells in the thoracic duct, circulating GC-like Tfh cells, and circulating non-GC Tfh cells

\begin{tabular}{|c|c|c|c|c|}
\hline Marker & $\begin{array}{l}\text { Lymph node } \\
\text { GC Tfh cells }{ }^{\mathrm{A}}\end{array}$ & $\begin{array}{l}\text { Thoracic duct } \\
\mathrm{Br} / \mathrm{Br} \text { Tfh cells }\end{array}$ & $\begin{array}{l}\text { Circulation } \\
\text { GC cTfh cells }\end{array}$ & $\begin{array}{c}\text { Circulation } \\
\text { non-GC cTfh cells }\end{array}$ \\
\hline CXCR5 & ++ & ++ & + & + \\
\hline PD-1 & ++ & ++ & + & $+1-$ \\
\hline ICOS & ++ & ++ & + & $+1-$ \\
\hline CD38 & ++ & ++ & + & $+1-$ \\
\hline IL-21 & +++ & ++ & + & + \\
\hline CCR7 & - & ++ & $+1-$ & ++ \\
\hline SIPR1 & - & ++ & + & + \\
\hline BCL6 & ++ & + & $+/-$ & - \\
\hline
\end{tabular}

${ }^{A}$ Other markers that have been used for lymph node GC Tfh cells include CD57, CD200, and CXCR4. ${ }^{B}$ Marker combinations for GC cTfh cells commonly used include PD-1+ICOS+CD38+ $(12,14), \mathrm{PD}^{+} 1^{+} \mathrm{ICOS}^{+}$ (17), and PD-1 $1^{\text {hi }} C C R 7^{10}(18)$ after gating on nonnaive $C D 4^{+} T$ cells, total $C X C R 5^{+} C D 4^{+} T$ cells, and memory CXCR5 ${ }^{+} \mathrm{CD}^{+}{ }^{+} \mathrm{T}$ cells, respectively. 'Markers used to identify GC-like cTfh cells by Vella et al. in this issue (12) and previously (14) by the same group.

turned to an alternate GC-like cTfh phenotype in blood, the $\mathrm{ICOS}^{+} \mathrm{CD}_{3} 8^{+}$subset, which was more abundant than the $\mathrm{Br} / \mathrm{Br}$ phenotype. Previously, this group had demonstrated recurrent oligoclonal expansions of the $\mathrm{ICOS}^{+} \mathrm{CD} 8^{+} \mathrm{cTfh}$ cell subset 7 days after successive yearly influenza vaccinations in volunteers (14, 15). Here, they show transcriptional and epigenetic similarity of $\mathrm{ICOS}^{+} \mathrm{CD} 38^{+}$cTfh cells to thoracic duct $\mathrm{Br} / \mathrm{Br}$ Tfh cells and SLO GC Tfh cells as well as higher S1PR1 expression than ICOS $^{-}$CD $38^{-}$cTfh cells. With these observations, the study comes close to establishing ties of $\mathrm{ICOS}^{+} \mathrm{CD} 38^{+}$ cTfh cells with SLO GC Tfh cells; however, a direct comparison of $\mathrm{CD}^{+} 8^{+} \mathrm{ICOS}^{+} \mathrm{Tfh}$ cells in each of the three compartments would have clinched this relationship.

\section{Concluding remarks}

This study is important in that it has provided the best link so far between Tfh cells in lymph nodes, thoracic duct, and peripheral blood; a key contribution is the direct examination in humans of Tfh cells in thoracic duct compared with those in lymph nodes and blood. A burning question has been whether the c $\mathrm{Tfh}$ cell pool contains an identifiable GC-experienced subset. The field is somewhat complex, and several studies have delineated cTfh and GC-like cTfh cell subsets with markers that overlap, but are not identical (Table 1 and refs. 4, 14, 16-21). Heterogeneity of cTfh subsets, transient marker expression, or dependence on antigen pose challenges in this context.
The importance of PD-1 in GC Tfh cels is unquestionable for SLO, but its role in cTfh cells is unclear and basal CD38 expression decreases with age (22). Are there minimal essential criteria for classifying Tfh cells in circulation? CXCR5 expression in memory $\mathrm{CD}^{+} \mathrm{T}$ cells with IL-21 production capability may suffice as overall cTfh cell markers. For GC cTfh cells, ICOS expression appears critical even in a quiescent state. Stimulus-induced CD4OL or equivalent markers demonstrate antigen specificity $(23,24)$. Now may be an opportune time to perform quantitative and qualitative investigations in vaccination/infection protocols in the context of age and clinical states to arrive at a unifying consensus. At the same time, assessing B cell helper function is critical. A plasmablast response (such as occurs on day seven after immunization) is unparalleled as an indicator of GC activity (25). The identification of a true GCderived cTfh cell subset is a highly desirable goal for its utility as a biomarker to monitor lymphoid GC Tfh cell activity and as a therapeutic target. The study by Vella et al. has paved the way for future research of cTfh cells in health and disease.

\section{Acknowledgments}

SP is supported by NIH grants AI108472, AI123048, AI127347, AI142669 and P30AI073961. Discussion with lab members and colleagues is greatly appreciated.

Address correspondence to: Savita Pahwa, Department of Microbiology and Immu- nology, University of Miami Miller School of Medicine, 1580 NW 10th Avenue; BCRI 712, Miami, Florida 33136, USA. Phone: 305.243.7732; Email: spahwa@ med.miami.edu.

1. Crotty S. Follicular helper CD4 T cells (TFH). Annu Rev Immunol. 2011;29:621-663.

2. Vinuesa CG, Linterman MA, Yu D, MacLennan IC. Follicular helper T cells. Annu Rev Immunol. 2016;34:335-368.

3. Pauthner $\mathrm{M}$, et al. Elicitation of robust tier 2 neutralizing antibody responses in nonhuman primates by HIV envelope trimer immunization using optimized approaches. Immunity. 2017;46(6):1073-1088.e6.

4. Morita R, et al. Human blood CXCR5(+) CD4(+) T cells are counterparts of $\mathrm{T}$ follicular cells and contain specific subsets that differentially support antibody secretion. Immunity. 2011;34(1):108-121.

5. Schmitt N, Bentebibel SE, Ueno H. Phenotype and functions of memory Th cells in human blood. Trends Immunol. 2014;35(9):436-442.

6. Song W, Craft J. T follicular helper cell heterogeneity: Time, space, and function. Immunol Rev. 2019;288(1):85-96.

7. Gowans JL, Knight EJ. The route of re-circulation of lymphocytes in the rat. Proc R Soc Lond, B, Biol Sci. 1964;159:257-282.

8. Girard JP, Moussion C, Förster R. HEVs, lymphatics and homeostatic immune cell trafficking in lymph nodes. Nat Rev Immunol. 2012;12(11):762-773.

9. Cyster JG, Schwab SR. Sphingosine-1-phosphate and lymphocyte egress from lymphoid organs. Anпи Rev Immunol. 2012;30:69-94.

10. Lian J, Luster AD. Chemokine-guided cell positioning in the lymph node orchestrates the generation of adaptive immune responses. Curr Opin Cell Biol. 2015;36:1-6.

11. Suzuki K, Hayano Y, Nakai A, Furuta F, Noda M. Adrenergic control of the adaptive immune response by diurnal lymphocyte recirculation through lymph nodes. JExp Med. 2016;213(12):2567-2574.

12. Vella $\mathrm{LA}$, et al. $\mathrm{T}$ follicular helper cells in human efferent lymph retain lymphoid characteristics. JClin Invest. 2019;129(8):3185-3200.

13. Beura LK, et al. T cells in nonlymphoid tissues give rise to lymph-node-resident memory $\mathrm{T}$ cells. Immunity. 2018;48(2):327-338.e5.

14. Herati RS, et al. Successive annual influenza vaccination induces a recurrent oligoclonotypic memory response in circulating $\mathrm{T}$ follicular helper cells. Sci Immunol. 2017;2(8):eaag2152.

15. Herati RS, et al. Circulating CXCR5+PD-1+ response predicts influenza vaccine antibody responses in young adults but not elderly adults. J Immunol. 2014;193(7):3528-3537.

16. Locci M, et al. Human circulating PD-1+ CXCR3-CXCR5+ memory Tfh cells are highly functional and correlate with broadly neutralizing HIV antibody responses. Immunity. 2013;39(4):758-769.

17. Heit A, et al. Vaccination establishes clonal relatives of germinal center $\mathrm{T}$ cells in the blood of 
humans. J Exp Med. 2017;214(7):2139-2152.

18. He J, et al. Circulating precursor CCR7(lo)PD-1(hi) $\mathrm{CXCR}^{+} \mathrm{CD} 4^{+} \mathrm{T}$ cells indicate $\mathrm{Tfh}$ cell activity and promote antibody responses upon antigen reexposure. Immunity. 2013;39(4):770-781.

19. Bentebibel SE, et al. ICOS(+)PD-1(+)CXCR3(+) $\mathrm{T}$ follicular helper cells contribute to the generation of high-avidity antibodies following influenza vaccination. Sci Rep. 2016;6:26494.

20. Pallikkuth S, et al. Impaired peripheral blood
T-follicular helper cell function in HIV-infected nonresponders to the $2009 \mathrm{H1N1} / 09$ vaccine. Blood. 2012;120(5):985-993.

21. Moysi E, et al. Altered immune cell follicular dynamics in HIV infection following influenza vaccination. J Clin Invest. 2018;128(7):3171-3185

22. de Armas LR, et al. Reevaluation of immune activation in the era of CART and an aging HIV-infected population. JCI Insight. 2017;2(20):e95726. 23. Pallikkuth S, et al. Dysfunctional peripheral
$\mathrm{T}$ follicular helper cells dominate in people with impaired influenza vaccine responses: Results from the FLORAH study. PLoS Biol. 2019;17(5):e3000257.

24. Reiss S, et al. Comparative analysis of activation induced marker (AIM) assays for sensitive identification of antigen-specific CD4 T cells. PLoS ONE. 2017;12(10):e0186998.

25. De Silva NS, Klein U. Dynamics of B cells in germinal centres. Nat Rev Immunol. 2015;15(3):137-148. 